\title{
BMJ Open Endovenous ablation and surgery in great saphenous vein reflux: a systematic review and network meta-analysis of randomised controlled trials protocol
}

\author{
Boonying Siribumrungwong, ${ }^{1,2}$ Kanoklada Srikuea, ${ }^{1}$ Saritphat Orrapin, ${ }^{1}$ \\ Thoetphum Benyakorn, ${ }^{1}$ Kittipan Rerkasem, ${ }^{3}$ Ammarin Thakkinstian ${ }^{4}$
}

To cite: Siribumrungwong B, Srikuea K, Orrapin S, et al. Endovenous ablation and surgery in great saphenous vein reflux: a systematic review and network meta-analysis of randomised controlled trials protocol. BMJ Open 2019;9:e024813. doi:10.1136/ bmjopen-2018-024813

- Prepublication history for this paper is available online. To view these files, please visit the journal online (http://dx.doi org/10.1136/bmjopen-2018024813).

Received 14 June 2018 Revised 20 November 2018 Accepted 3 December 2018

Check for updates

(C) Author(s) (or their employer(s)) 2019. Re-use permitted under CC BY-NC. No commercial re-use. See rights and permissions. Published by BMJ.

For numbered affiliations see end of article.

Correspondence to Dr Ammarin Thakkinstian; ammarin.tha@mahidol.ac.th

\section{ABSTRACT}

Introduction Endovenous ablations are the new standard procedures for treatment of great saphenous vein reflux including endovenous laser ablation (EVLA), radio frequency ablation (RFA), endovenous steam ablation (EVSA), mechanochemical ablation (MOCA), cyanoacrylate injection and ultrasound-guided foam sclerotherapy (UGFS). EVLA and RFA have demonstrated similar anatomical success for short-term outcome, but results are controversial for longer term ( $\geq 5$ years). Additional evidences from randomised controlled trials have been published. This study is, therefore, conducted to, directly and indirectly, compare outcomes among all procedures stratifying by short-term and long-term follow-up. Methods and analysis Medline and Scopus will be searched from 2000 to September 2018 with predefined search strategy. Interventions of interest are open surgery (ie, saphenofemoral or high ligation (HL) with stripping) and endovenous ablations (ie, EVLA, RFA, EVSA, MOCA, cyanoacrylate injection and UGFS). The primary outcome is anatomical success. Two independent reviewers will select studies, extract data and assess risk of bias. Disagreement will be adjudicated by the third party. Outcomes will be directly pooled if there are at least three studies in that comparison. A fixed-effect model will be used unless heterogeneity is present, in which case a random-effect model will be applied. Sources of heterogeneity will be explored using meta-regression analysis, and sub-group analysis will be done accordingly. Publication bias will be assessed using Egger's test and funnel plot. A network meta-analysis will be applied to indirect compare all interventions including RFA, EVLA, EVLA with HL, UGFS, UGFS with $H L$ and $H L$ with stripping. Probability of being best intervention will be estimated and ranked. Inconsistency assumption will be checked using a designby-treatment interaction model.

Ethics and dissemination Ethical approval is not required for systematic review and network meta-analysis. The study will be published in a peer-reviewed journal. PROSPERO registration number CRD42018096794.

\section{INTRODUCTION}

Chronic venous disease is a common condition, which affects both men and women with the prevalence rate of $30 \%-50 \% .^{12}$ This has

\section{Strengths and limitations of this study}

- This review will include all relevant randomised controlled trials comparing outcomes between endovenous ablation and surgery from 2000 to September 2018.

- Stratified analysis by short-term and long-term follow-up will be performed.

- Probability of being the best procedure will be estimated and provided.

led to significant health spending, and about $1 \%-2 \%$ of healthcare budgets have been spent for venous disease in European countries. ${ }^{3}$ Great saphenous vein (GSV) reflux is the most common site of reflux accounting for about $80 \%$ of all reflux sites. ${ }^{4}$ GSV ablation is recommended to improve symptoms and quality of life of patients. ${ }^{56}$

To ablate GSV, endovenous ablations are recommended over surgery as a new standard treatment. ${ }^{6}$ The benefits over open surgery are less postoperative pain, a lower rate of surgical site infection, faster return to normal activities and work. ${ }^{7}$ However, they are accompanied by higher equipment costs. ${ }^{8}$ Therefore, many techniques of endovenous ablation have emerged including endovenous laser ablation (EVLA), radio frequency ablation (RFA), endovenous steam ablation (EVSA) and ultrasound-guided foam sclerotherapy (UGFS). Two novel techniques of non-tumescent non-thermal endovenous ablation (NTNT) including mechanochemical ablation (MOCA) and cyanoacrylate injection have been introduced for several years with promising early results. ${ }^{9}$

Directly related outcome after GSV ablation is an anatomical success, which is patency of the GSV after ablation. However, failure or recurrence cannot be avoided which is classified as technical failure, periprocedural, 
early, mid-term and late failure when it occurs $\leq 3$ days, $\leq 1$ month, 1year, 1-3 years and $>3$ years after the operation. Sources of recurrences could be neovascularisation and reflux in tributaries in which the former might occur more after open surgery whereas the latter often occurs after endovenous ablation without high ligation (HL). ${ }^{10}{ }^{11}$ Another important outcome is patient-reported outcome measurements, which measures patients' perspective in both generic and specific quality of life. ${ }^{6}$

Previous evidences about efficacy of these procedures had been pooled considering short-term to long-term outcomes. The first systematic review in 2012 included 28 randomised controlled trials (RCTs) to compare shortterm/mid-term outcomes of endovenous procedures with surgery. It found benefits of endovenous procedures (ie, EVLA, RFA and UGFS) over open surgery in postoperative pain, morbidity and faster recovery with similar efficacies for EVLA and RFA, but less efficacy for UGFS. ${ }^{7}$ Two other systematic reviews in $2017^{10}$ and $2018^{11}$ considered only long-term outcomes by including 12 and 9 RCTs with $>5$ years follow-up, respectively. Although the former meta-analysis ${ }^{12}$ considered only RCTs, they pooled outcome data (ie, success/recurrent reflux rates and mean difference (MD)) comparing before versus after of each intervention without directly comparing these outcomes between groups. As a result, randomisation may be broken and thus bias the results. The latest meta-analysis ${ }^{11}$ could not detect whether recurrence rates between EVLA, RFA and surgery were different due to small numbers of included studies and subjects.

Some additional RCTs comparing endovenous procedures and open surgery or comparing among endovenous ablations have been published later with varying follow-up times and also surgical techniques, that is, with or without HL. ${ }^{13-26}$ In addition, RCTs comparing among endovenous techniques including NTNT (ie, EVSA, MOCA and cyanoacrylate injection) have also been published. These data have not yet been updated in the aforementioned meta-analyses with long-term outcomes. In addition, accurate and precise magnitude of benefit of endovenous procedures over surgery along time horizon of treatment is important for economic analysis. ${ }^{27}$ Therefore, this systematic review and network meta-analysis is conducted which aims to, directly and indirectly, compare clinical outcomes between interventions stratifying by the time of follow-up including anatomical success, clinical recurrence and quality of life. Postoperative outcomes including postoperative pain, time to return to normal activities and work, and complications (ie, wound infection, haematoma, paresthesia, ecchymosis and deep venous thrombosis) will be pooled using all available data. In addition, source of recurrences (ie, neovascularisation and reflux in tributaries) and reintervention rates will also be pooled. A probability of being the best intervention will be estimated and ranked for each outcome. Risk and benefit will be then compared.

\section{METHODS}

The protocol was developed according to Preferred Reporting Items for Systematic Reviews and Meta-analyses statement for reporting systematic reviews and meta-analyses ${ }^{28}$ and extension statement for systematic reviews incorporating network meta-analyses of healthcare interventions. $^{29}$

\section{Search strategy}

Medline and Scopus will be searched from 2000 to September 2018, and will be updated every 3 months until August 2019. Search terms are constructed according to patients and intervention/comparator as follows: 'Varicose veins' [Mesh] OR 'Saphenous vein' [Mesh] OR varicose OR saphenous NOT esophageal; radiofrequency OR RFA OR VNUS OR endovenous OR EVLT OR EVLA OR laser OR sclerotherapy[Mesh] OR foam sclerotherapy OR UGFS OR stripping OR sapheno-femoral ligation OR surgery OR steam OR glue OR cyanoacrylate OR clarivein OR mechanochemical OR mechano-chemical. These search terms of the two domains will be combined with AND with limited to clinical trial, human and English articles. Reference lists from previous meta-analysis and all eligible papers will be reviewed for relevant studies.

\section{Study selection}

Study selection will be done by two independent authors (BS and TB). Title and abstract will be screened for eligible criteria, the full text will then be reviewed if a decision cannot be made. Any disagreement will be adjudicated by a third party (AT). Data from multiple publication studies will be combined as one publication for analysis.

RCTs studied in patients with GSV reflux will be included if they meet the following criteria: had any pair of the following interventions including endovenous thermal ablation (ie, EVLA, RFA and EVSA), endovenous non-thermal non-tumescent ablations (ie, MOCA, UGFS, cyanoacrylate injection) and surgery; had at least one following outcomes including anatomical success, postoperative pain, wound infection, haematoma, ecchymosis, deep vein thrombosis, time to return to normal activities and work, clinical recurrence and quality of life. Studies will be excluded if they have insufficient data for pooling.

\section{Interventions}

Interventions of interest are open surgery (ie, saphenofemoral or HL with stripping of the GSV), endovenous thermal ablations (ie, EVLA, RFA and EVSA) and non-thermal non-tumescent endovenous ablation (ie, UGFS, MOCA, cyanoacrylate injection). EVLA ablates GSV using laser energy with varying wavelength such as $810,940,980,1470$ and $1560 \mathrm{~nm} .{ }^{30}{ }^{31}$ RFA generates heat by radio frequency energy. Both RFA and EVLA are usually performed with tumescent anaesthesia to prevent thermal injury to adjacent tissue. EVSA uses heat from steam to ablate GSV. MOCA both mechanically and chemically injured endothelium of targeted vein. 
Cyanoacrylate is polymerised into solid form to occlude vein after injection. UGFS damages endothelium causing occlusion of the vein, which is injected to the GSV by either direct puncture or via a catheter. ${ }^{32}$ Foam sclerosant can be developed manually or by the manufacture. HL might or might not be applied with endovenous procedures. Comparison of interests will be any pair among different types of ablations or the same type of ablations, but different techniques (eg, different sclerosants and/ or concentrations for UGFS, short vs long wavelengths or pull back types for laser), if data are sufficient for pooling and there are common comparators in the network mapping.

\section{Outcomes}

The primary outcome of interest is anatomical success, which was originally defined as incomplete stripping for open surgery and non-occlusion of GSV with or without reflux diagnosed by a duplex scan. This outcome will be considered according to the time frame of follow-up, that is, periprocedural, early, mid-term and late failure. ${ }^{6}$

Secondary outcomes of interest are clinical recurrence, postoperative pain, time to return to normal activities and work, self-reported quality of life, reintervention rate and postoperative complications (ie, haematoma, ecchymosis, paresthesia and deep vein thrombosis). Quality of life will be compared according to the time frame of follow-up. Clinical recurrence will be defined as clinical detected recurrence of varicose vein. Neovascularisation and reflux in tributaries will also be extracted and compared.

\section{Data extraction}

Two independent authors (BS and KS) will extract data using standardised data extraction forms. General characteristics of studies and interventions including patients' severity, age, details of the intervention, duration of follow-up, type of anaesthesia, compression method, tumescent anaesthesia, primary outcome definition, concomitant phlebectomy and sclerotherapy will be extracted. These data will be used for exploring the source of heterogeneity. Mean (SD) and frequencies of outcomes data by intervention will be extracted for pooling. MD or risk ratio (RR) will be used in case of no summary data provided in the study. Inconsistent data will be solved by consensus with a third party (AT) and finalised. An author will contact corresponding authors twice for missing data.

\section{Risk of bias assessment}

Studies will be assessed for risk of bias using Cochrane Collaboration's tool ${ }^{33}$ by two independent researchers (BS and SO). This tool consists of seven domains: random sequence generation, allocation concealment, blinding of participants and personnel, blinding of outcome assessors, incomplete outcome reports, selective outcome reports and other source of bias. Disagreement will be resolved by a third party (AT).

\section{Grading evidence}

Quality of evidence will be graded separately for each outcome using a tool suggested by the Grading of
Recommendations Assessment, Development and Evaluation (GRADE) Working Group. ${ }^{34}$ Five domains will be assessed including study limitations, consistency, indirectness, imprecision and publication bias. The evidence will be downgraded one and two levels for serious and very serious concerns, respectively.

\section{Statistical analysis}

\section{Direct meta-analysis}

Data will be directly pooled if there are at least three studies for each comparison. RRs with $95 \%$ CI will be estimated for a dichotomous outcome. A continuity correction will be used if there is a zero cell. The RRs will be pooled using inverse variance, and Dersimonian and Laird for data without and with heterogeneity, respectively. For continuous outcome, unstandardised or standardised MD with 95\% CI will be estimated and pooled across studies if outcome measures are the same and different scales, respectively. Mean and SD will be estimated from median and range/interquartile, if a study did not report mean and SD. ${ }^{35}$

Heterogeneity will be assessed using a degree of heterogeneity $\left(\mathrm{I}^{2}\right)$ and $Q$ test. If either $\mathrm{I}^{2} \geq 25 \%$ or $Q$ test is significant with $\mathrm{p}<0.10$, the results will be considered as heterogeneous and random-effect model will be applied. Possible source of heterogeneity will be explored by fitting studies' characteristics (ie, concomitant phlebectomy or foam sclerotherapy, type of anaesthesia and compression method), different of interventional techniques in each type of endovenous ablation (ie, laser wavelength, catheter use in UGFS, and type of sclerosant and concentration), outcomes definition (ie, non-occlusion, non-occlusion with or without reflux) and patient's characteristics (ie, age, severity) in a meta-regression model, if data are sufficient. Subgroup or sensitivity analysis will be performed accordingly to factors that can reduce the degree of heterogeneity. Publication bias will be assessed by funnel plot and Egger test. If there is evidence of asymmetry of the funnel by either of these two, a contour-enhanced plot will be constructed to distinguish whether a source of asymmetry is due to heterogeneity or missing studies.

\section{Network meta-analysis}

A network meta-analysis ${ }^{36}$ will be performed to indirectly compare among interventions including RFA, EVLA, EVLA with HL, UGFS, UGFS with HL, EVSA, MOCA, cyanoacrylate injection and HL with stripping. HL with stripping will be used as a common comparator. The analysis will be performed by the following steps: first, relative intervention effect, that is, RR along with its variancecovariance will be estimated by binary regression analysis. A multivariate random-effect meta-analysis with consistency mode will then be used to pool RRs across studies. Mixed intervention comparisons will be next estimated. A probability of being the best intervention will be estimated and ranked using surface under the cumulative ranking method, and rankogram will be plotted accordingly. Cluster rank plot will be constructed by comparing the probability of being risk and benefit. 
The inconsistency assumption (ie, whether direct effects agree with the indirect effects) will be checked using a design-by-treatment interaction model. If this assumption is not met, an inconsistency factor (ie, $\ln (\mathrm{R}$ Rdirect)-ln(RRindirect)) will be estimated and tested. In addition, a comparison-adjusted funnel plot taking into account different comparisons will be plotted to explore whether there is evidence of small study effect for the whole network. ${ }^{36} 37$

Analyses will be performed using STATA V.15.0. A $\mathrm{p}<0.05$ will be considered as statistically significant, except heterogeneity test where $\mathrm{p}<0.10$ will be used.

\section{Patient and public involvement}

Patients and public will not be involved in this study.

\section{Ethics and dissemination}

Results of the study will be presented in international meeting. The manuscript will be submitted to peer-reviewed journal.

\section{Author affiliations}

${ }^{1}$ Division of Vascular and Endovascular Surgery, Department of Surgery, Faculty of Medicine, Thammasat University Hospital, Pathum Thani, Thailand

${ }^{2}$ Center of Excellence in Applied Epidemiology, Faculty of Medicine, Thammasat University Hospital, Pathum Thani, Thailand

${ }^{3}$ NCD Center, RIHES and Department of Surgery, Faculty of Medicine, Chiang Mai University, Chiang Mai, Thailand

${ }^{4}$ Section for Clinical Epidemiology and Biostatistics, Faculty of Medicine,

Ramathibodi Hospital, Mahidol University, Bangkok, Thailand

Contributors BS initiated research question design review methods and wrote the protocol. KS wrote and registered protocol. SO wrote and registered protocol. TB wrote and commented on protocol. KR wrote and commented on protocol. AT designed review methods, wrote and commented on protocol.

Funding The authors have not declared a specific grant for this research from any funding agency in the public, commercial or not-for-profit sectors.

Competing interests None declared.

Patient consent for publication Not required

Ethics approval Ethical consideration and ethic committee approval are not required from the nature of systematic review and network meta-analysis.

Provenance and peer review Not commissioned; externally peer reviewed.

Open access This is an open access article distributed in accordance with the Creative Commons Attribution Non Commercial (CC BY-NC 4.0) license, which permits others to distribute, remix, adapt, build upon this work non-commercially, and license their derivative works on different terms, provided the original work is properly cited, appropriate credit is given, any changes made indicated, and the use is non-commercial. See: http://creativecommons.org/licenses/by-nc/4.0/.

\section{REFERENCES}

1. Carpentier PH, Maricq HR, Biro C, et al. Prevalence, risk factors, and clinical patterns of chronic venous disorders of lower limbs: a population-based study in France. J Vasc Surg 2004;40:650-9.

2. Beebe-Dimmer JL, Pfeifer JR, Engle JS, et al. The epidemiology of chronic venous insufficiency and varicose veins. Ann Epidemiol 2005;15:175-84.

3. Ruckley CV. Socioeconomic impact of chronic venous insufficiency and leg ulcers. Angiology 1997;48:67-9.

4. Kanchanabat B, Stapanavatr W. Venous ultrasonography findings and clinical correlations in 104 Thai patients with chronic venous insufficiency of the legs. Singapore Med J 2018;59:155-8.

5. Siribumrungwong B, Noorit P, Wilasrusmee C, et al. Quality of life after great saphenous vein ablation in Thai patients with great saphenous vein reflux. Asian J Surg 2017;40:295-300.
6. Gloviczki P, Comerota AJ, Dalsing MC, et al. The care of patients with varicose veins and associated chronic venous diseases: clinical practice guidelines of the Society for Vascular Surgery and the American Venous Forum. J Vasc Surg 2011:53(5 Suppl):2S-48.

7. Siribumrungwong $B$, Noorit $P$, Wilasrusmee $C$, et al. A systematic review and meta-analysis of randomised controlled trials comparing endovenous ablation and surgical intervention in patients with varicose vein. Eur J Vasc Endovasc Surg 2012;44:214-23.

8. Siribumrungwong $B$, Noorit $P$, Wilasrusmee $C$, et al. Cost-utility analysis of great saphenous vein ablation with radiofrequency, foam and surgery in the emerging health-care setting of Thailand. Phlebology 2016;31:573-81.

9. Vos CG, Ünlü Ç, Bosma J, et al. A systematic review and metaanalysis of two novel techniques of nonthermal endovenous ablation of the great saphenous vein. J Vasc Surg Venous Lymphat Disord 2017;5:880-96.

10. Disselhoff BC, der Kinderen DJ, Kelder JC, et al. Five-year results of a randomised clinical trial of endovenous laser ablation of the great saphenous vein with and without ligation of the saphenofemoral junction. Eur J Vasc Endovasc Surg 2011;41:685-90.

11. Theivacumar NS, Darwood R, Gough MJ. Neovascularisation and recurrence 2 years after varicose vein treatment for saphenofemoral and great saphenous vein reflux: a comparison of surgery and endovenous laser ablation. Eur J Vasc Endovasc Surg 2009;38:203-7.

12. Kheirelseid EAH, Crowe G, Sehgal R, et al. Systematic review and meta-analysis of randomized controlled trials evaluating long-term outcomes of endovenous management of lower extremity varicose veins. J Vasc Surg Venous Lymphat Disord 2018;6:256-70.

13. Rass K, Frings N, Glowacki P, et al. Comparable effectiveness of endovenous laser ablation and high ligation with stripping of the great saphenous vein: two-year results of a randomized clinical trial (RELACS study). Arch Dermatol 2012;148:49-58.

14. Basela $\mathrm{H}$, Aydina $\mathrm{C}$, Aya $\mathrm{Y}$, et al. Endovenous laser ablation (EVLA) versus high ligation and striping (HL/S): Two-years follow up. Eastern Journal of Medicine 2012;17:83-7.

15. Flessenkämper I, Hartmann M, Stenger D, et al. Endovenous laser ablation with and without high ligation compared with high ligation and stripping in the treatment of great saphenous varicose veins: initial results of a multicentre randomized controlled trial. Phlebology 2013;28:16-23.

16. Mozafar $\mathrm{M}$, Atqiaee $\mathrm{K}$, Haghighatkhah $\mathrm{H}$, et al. Endovenous laser ablation of the great saphenous vein versus high ligation: long-term results. Lasers Med Sci 2014;29:765-71.

17. Shadid N, Ceulen R, Nelemans P, et al. Randomized clinical trial of ultrasound-guided foam sclerotherapy versus surgery for the incompetent great saphenous vein. Br J Surg 2012;99:1062-70.

18. Yin $\mathrm{H}, \mathrm{He} \mathrm{H}$, Wang $\mathrm{M}$, et al. Prospective randomized study of ultrasound-guided foam sclerotherapy combined with great saphenous vein high ligation in the treatment of severe lower extremity varicosis. Ann Vasc Surg 2017;39:256-63.

19. Venermo M, Saarinen J, Eskelinen E, et al. Randomized clinical trial comparing surgery, endovenous laser ablation and ultrasoundguided foam sclerotherapy for the treatment of great saphenous varicose veins. Br J Surg 2016;103:1438-44.

20. Brittenden J, Cotton SC, Elders A, et al. A randomized trial comparing treatments for varicose veins. N Engl J Med 2014;371:1218-27.

21. Biemans AA, Kockaert M, Akkersdijk GP, et al. Comparing endovenous laser ablation, foam sclerotherapy, and conventional surgery for great saphenous varicose veins. J Vasc Surg 2013:58:727-34.

22. Mendes CA, Martins AA, Fukuda JM, et al. Randomized trial of radiofrequency ablation versus conventional surgery for superficial venous insufficiency: if you don't tell, they won't know. Clinics 2016;71:650-6.

23. Sydnor M, Mavropoulos J, Slobodnik N, et al. A randomized prospective long-term ( $>1$ year) clinical trial comparing the efficacy and safety of radiofrequency ablation to $980 \mathrm{~nm}$ laser ablation of the great saphenous vein. Phlebology 2017;32:415-24.

24. Bozoglan O, Mese B, Eroglu E, et al. Comparison of endovenous laser and radiofrequency ablation in treating varicose veins in the same patient. Vasc Endovascular Surg 2016;50:47-51.

25. Mese B, Bozoglan O, Eroglu E, et al. A Comparison of 1,470-nm endovenous laser ablation and radiofrequency ablation in the treatment of great saphenous veins $10 \mathrm{~mm}$ or more in size. Ann Vasc Surg 2015;29:1368-72.

26. Nordon IM, Hinchliffe RJ, Brar R, et al. A prospective double-blind randomized controlled trial of radiofrequency versus laser treatment of the great saphenous vein in patients with varicose veins. Ann Surg 2011;254:876-81. 
27. Permsuwan U, Guntawongwan K, Buddhawongsa P. Handling time in economic evaluation studies. J Med Assoc Thai 2014;97 Suppl 5(Suppl 5):S50-8.

28. Liberati A, Altman DG, Tetzlaff J, et al. The PRISMA statement for reporting systematic reviews and meta-analyses of studies that evaluate healthcare interventions: explanation and elaboration. BMJ 2009;339:b2700.

29. Hutton B, Salanti G, Caldwell DM, et al. The PRISMA extension statement for reporting of systematic reviews incorporating network meta-analyses of health care interventions: checklist and explanations. Ann Intern Med 2015;162:777-84.

30. Cavallini A. Endovenous laser treatment of saphenous veins: is there clinical difference using different endovenous laser wavelenghts? Int Angiol 2015;34:1-8.

31. Galanopoulos G, Lambidis C. Minimally invasive treatment of varicose veins: Endovenous laser ablation (EVLA). Int J Surg 2012;10:134-9.
32. van Eekeren RR, Boersma D, de Vries JP, et al. Update of endovenous treatment modalities for insufficient saphenous veins-a review of literature. Semin Vasc Surg 2014;27:118-36.

33. Higgins JP, Altman DG, Gøtzsche PC, et al. The cochrane collaboration's tool for assessing risk of bias in randomised trials. BMJ 2011;343:d5928.

34. Puhan MA, Schünemann HJ, Murad MH, et al. A GRADE Working Group approach for rating the quality of treatment effect estimates from network meta-analysis. BMJ 2014;349:g5630.

35. Hozo SP, Djulbegovic B, Hozo I. Estimating the mean and variance from the median, range, and the size of a sample. BMC Med Res Methodol 2005;5:13.

36. White IR. Network Meta-analysis. Stata J 2015;15:951-85.

37. Chaimani A, Salanti G. Using network meta-analysis to evaluate the existence of small-study effects in a network of interventions. Res Synth Methods 2012;3:161-76. 